\title{
Numerical Imitation of the Synchronization of Gene Repressilators
}

\author{
$\mathrm{Yu} \mathrm{Wu}$ \\ Cambridge A-Level Center \\ Wuxi No.1 High School \\ Wuxi, China
}

\author{
Junhui Gao \\ Shanghai Center for Bioinformation Technology \\ Shanghai, China
}

\begin{abstract}
Jordi Garcia-Ojalvo and his partners published an article on PNS in 2004, building numerical models of synchronization of many gene oscillators. We got the examples of the process of synchronization by imitating the model with numerical value and studied on the relationship between the value of $Q$, the number of cells and the process of synchronization. We sketched a graph and got a result that are contradict to what they mentioned in the article.
\end{abstract}

Keywords-gene oscillators, process of synchronization; numerical imitation; value of $Q$

\section{INTRODUCTION}

Gene oscillators tend to be synchronized within numbers of cells because of both the extinct driving from the environment and the intrinsic rhythms generated by specialized cellular clocks within the organism itself. In article [1], Jordi Garcia-Ojalvo and his partners studied on the change of dynamic models of mRNA in cells and the concentration of protein as time changed. We got the examples of the process of synchronization by imitating the model with numerical value and studied on the relationship between the value of $\mathrm{Q}$, the number of cells and the process of synchronization.

\section{METHODS}

\section{A. Numerical Imitation}

Figure 1 expresses three proteins and mRNA associated with AI of the corresponding genes in cells.

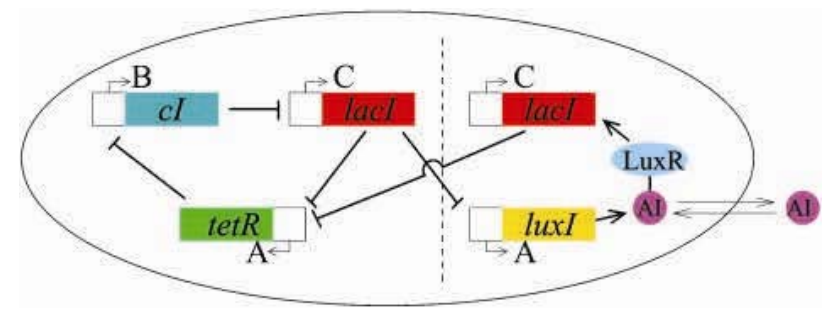

FIGURE I. SCHEME OF THE REPRESSILATORS NETWORK COUPLED TO A QUORUM SENSING MECHANISM. THE ORIGINAL REPRESSILATOR MODULE IS LOCATED AT THE LEFT OF THE

VERTICAL DASHED LINE, AND THE NEW COUPLING MODULE APPEARS AT THE RIGHT. THE LETTERS A, B, AND C CORRESPOND TO THE NOTATION USED IN THE TEXT. THE COUPLING MODULE CAN BE ADDED TO EXISTING REPRESSILATOR STRAINS.
The dynamic equations of the three mRNA:

$$
\begin{gathered}
\frac{d a_{i}}{d t}=-a_{i}+\frac{\alpha}{1+C_{i}^{n}}, \\
\frac{d b_{i}}{d t}=-b_{i}+\frac{\alpha}{1+A_{i}^{n}}, \\
\frac{d c_{i}}{d t}=-C_{i}+\frac{\alpha}{1+B_{i}^{n}}+\frac{\kappa S_{i}}{1+S_{i}}
\end{gathered}
$$

The dynamic equations of the three proteins:

$$
\begin{aligned}
\frac{d A_{i}}{d t} & =\beta\left(a_{i}-A_{i}\right), \\
\frac{d B_{i}}{d t} & =\beta\left(b_{i}-B_{i}\right), \\
\frac{d C_{i}}{d t} & =\beta\left(c_{i}-C_{i}\right)
\end{aligned}
$$

The concentration of AI is affected by degradation, synthesis, and diffusion of the TetR and LucI proteins. Assuming the equal lifetimes of the TetR and LucI proteins, they should have the same dynamic equations, and hence we will use the same variable to describe both protein concentrations. Consequently, the synthesis term of the AI rate equation will be proportionalto $A_{i}$. The concentrations of AI in the cell and the environment satisfy the equation:

$$
\frac{d S_{i}}{d t}=-k_{s e} S_{i}+k_{s 1} A_{i}-\eta\left(S_{i}-S_{e}\right)
$$

In which $\eta=\sigma A / V_{c} \equiv \delta / V_{c}$ measures the diffusion rate of AI through the cell membrane; $\sigma$ measures the permeability of the cell membrane; $V_{c}$ measures the volume of the cell; $k_{s 1}, k_{s 0}$ are constants; $S_{e}$ measures the concentration of AI inside the cell, which has the dynamic equation: 


$$
\frac{d S_{e}}{d t}=-k_{s e} S_{e}+\eta_{e x t} \sum_{j=1}^{N}\left(S_{j}-S_{e}\right)=-k_{s e} S_{e}+k_{d i f f}\left(\bar{S}-S_{e}\right)
$$

In the actual situation, we give $\dot{S}_{e}$ the value 0 , so that it has the equation:

$$
\begin{gathered}
S_{e}=\frac{k_{\text {diff }}}{k_{s e}+k_{d i f f}} \bar{S}=Q \bar{S} \\
Q=\frac{\delta N / V_{e x t}}{k_{s e}+\delta N / V_{e x t}}
\end{gathered}
$$

Q depends on the cell density $N /\left(V_{\text {ext }}+V_{c}\right) \approx N / V_{\text {ext }}$.

\section{B. The Results of the Numerical Imitation}

We adopted the dynamic equations of the proteins and mRNAs in the article, fixing the value of $\alpha, \beta, k_{s 0}, k_{s 1, N}$ and Q. Choosing 10 cells from random, we recorded the concentration of AI in every period. Finally, the 10 cells shown the same concentration, which represented the synchronization. The whole process is showed in Figure 2.

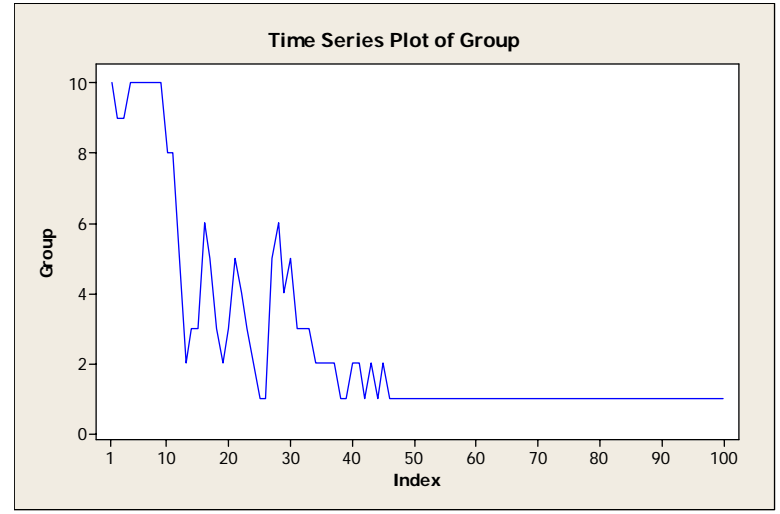

FIGURE II. THE EXPERIMENT PROCESS

In Figure 2, the abscissa axis represents the point in time

(30):10955-60. Epub 2004 Jul 15 (step number) while the vertical axis represents the number of groups that the concentration of AI divided in this period. As it can be seen in the graph, the number of groups reduced in the period from 0-50 with fluctuation; however, after the point 50 , there is only one group of concentration without fluctuation, which means that the cells are synchronized.

\section{DISCUSSION}

In the further step, we studied on the relationship between the value of $\mathrm{Q}$, the number of cells and the process of synchronization.

We chose different number of cells, represented by $\mathrm{N}$, 1000000, 500000, 100000, 50000, 10000, 5000 and different values of Q,0.8, 0.7, 0.6, 0.5, 0.4, 0.3, 0.2, 0.1. After repeating such process of numerical imitation and recording the time needed for synchronization, we got the results in Figure 3.

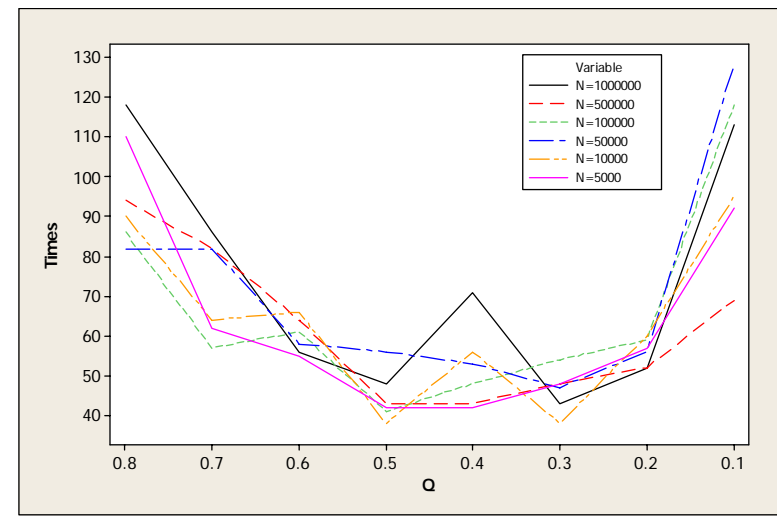

FIGURE III. THE PROCESS OF SYNCHRONIZATION WITH DIFFERENT VALUE OF N AND Q

In Figure 3 the abscissa axis represents the point in time (step number) while the vertical axis represents the time needed for reaching synchronization. The six lines with different colors display different values of $\mathrm{N}$. As it can be seen in the graph, the speed of the synchronization accelerated in period 0.8-0.5, reaching the maximum speed in period 0.5-0.3 and reduced in period 0.3-0.1.

\section{REFERENCE}

[1] Jordi Garcia-Ojalvo, Michael B. Elowitz, and Steven H. Strogatz Modeling a synthetic multicellular clock: Repressilators coupled by quorum sensing [J] PNAS2004 Jul 27;101 